\title{
A dual 3D DIC-system application for DSL strain and displacement measurements
}

\author{
I. Raurova, C. Berggreen ${ }^{\mathrm{a}}$ and R. Eriksen \\ Department of Mechanical Engineering, Technical University of Denmark, Nils Koppels Allé, 2800 \\ Kgs Lyngby, Denmark
}

\begin{abstract}
This paper describes a dual 3D Digital Image Correlation (DIC) system application for DLS strain and displacement measurements, where two 3D DIC-systems are used in parallel. The bonded specimens were tested to failure under monotonic loading in a uni-axial tensile testing machine at ambient temperature. Both surface inplane strain and full-field displacement values were recorded using two DIC systems: high speed (HS) and high resolution (HR). The HS system was used in a parallel setup with the HR system in order to detect the initial failure location and crack propagation rate during the brittle failure mechanism, where an interface crack is propagating between the straps and the inner adherent. Using two inherently different DIC systems involve a number of problems. This involves synchronization of the HS and HR systems, a common illumination level and speckle pattern. This paper therefore describes guidelines for a mutual system setup, applied in an experimental study of steel/epoxy DLS joints under pure tension.
\end{abstract}

\section{Introduction}

In the marine industry there is future potential for adhesives in various types of constructions which include super-structures for ships and offshore platforms as well as their application in connection with patch repair of cracks and corroded areas within the structure [1]. This technology is currently under consideration for repair of onboard floating production storage and off-loading units (FPSO's), where conventional repair by welding is very expensive due to safety [1]. A suitable and good representation of a patch repair of a steel structure is the DLS (Double Lap Shear) joint, which can be studied in 2D.

The aim of following paper is (a) to provide a quick guide to the application of a stereo 3D Digital Image Correlation (DIC) system setup to measure full-field 3D displacement and 2D strain fields at a specimen surface while undergoing testing, where two 3D DIC-systems are applied in parallel, (b) to describe the failure of the test specimens and finally (c) to shortly present results of the DLS-tests.

\footnotetext{
a e-mail : cbe@mek.dtu.dk
} 


\section{Experimental testing}

\subsection{Specimens}

The choice of suitable standard test specimens to characterize adhesive joints like a patch repair detail has been studied experimentally and analytically in the literature. A number of different specimens with various joint geometries and materials, adhesive properties, using tapering and stepping of adherents, have been proposed in the literature [1,2,6-9].

The joint geometry and stiffness/strength of the adhesive play an important role in achieving a high load capacity of the joint. Additionally, a proper and careful surface preparation of the adhesive interfaces before adhesion is a key for a strong adhesive joint.

\subsubsection{DSL joints specimens}

For this study, (a) 9 specimen types made from ultra high strength steel (UH) (b) 1 specimen type made from high strength steel $(\mathrm{H})$ and $(\mathrm{c}) 6$ specimen types made from mild steel (S) were manufactured. 1-3 replicas of all specimen types were manufactured. Figure 1 shows all specimen specifications and Table 1 includes dimensions and results only for specimens tested with DIC. The designations for the specimens specify "overlap length/strap thickness \& strap material/ number of batch + specimen \& fabrication site". For example a specimen designation of 100/3UH/D2.1 refers to $100 \mathrm{~mm}$ overlap / $3 \mathrm{~mm}$ thick ultra high strength steel straps / batch no.2, specimen no.1 fabricated at Technical University of Denmark (DTU) [D].

All specimens were made in 2 different strap thicknesses (3 and $6 \mathrm{~mm}$ ) and 5 different overlap lengths ${ }^{\mathrm{b}}(25,50,100,150$ and $200 \mathrm{~mm})$. The width of both the inner adherents and the straps is 25 $\mathrm{mm}$, whereas the nominal thickness of the adhesive layer is $0.5 \mathrm{~mm}$. Both magnitudes were kept constant for all specimens. The thickness of the inner adherents is $10 \mathrm{~mm}$ and common for nearly all specimens. The geometry as well as the general outline for the specimens, with the exception of the specimens 200/6UH/D9.1 and 200/6UH/D9.2, can be seen in Figure 1. The specimens 200/6UH/D9.1 and 200/6UH/D9.2 are special due to their double inner adherent.
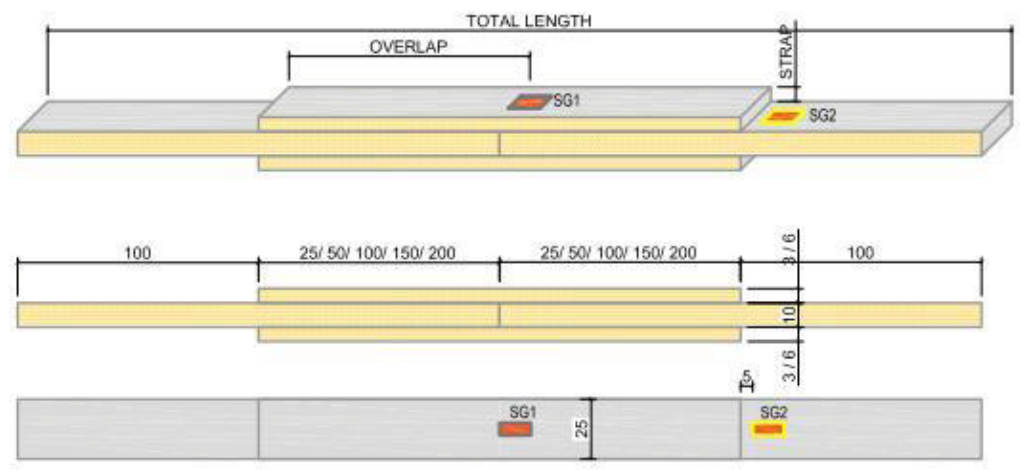

Fig. 1. Specimen geometry and strain gages positions.

The bonding process typically requires seven operations [2], this including surface roughening, degreasing, marking, adhesive application, positioning of clamps, curing and removal of clamps. The bonding surfaces were prepared by girt blasting. After the surface preparation, the outer adherents were glued using adhesive Araldite 2015 (two-part toughened epoxy adhesive) - evenly applied to all bonding surfaces. Finally to give a uniform thickness of the adhesive layer $(0.5 \mathrm{~mm})$, the clamps

\footnotetext{
${ }^{\mathrm{b}}$ Overlap length is defined as half of the total strap's length (see Figure 1)
} 
were tightened evenly and the specimens were cured in an oven at $85^{\circ} \mathrm{C}$ for 1 hour followed by a 2 hour cooling period at room temperature.

\subsection{Test and measurement equipment}

The DSL specimens were tested in a uni-axial $155 \mathrm{kN}$ MTS 810 servo-hydraulic testing machine equipped with a MTS $222 \mathrm{kN}$ load cell and hydraulic grips. Two DIC systems were used to measure 3D displacements and 2D strains at the specimen surface. Positioning of the DIC systems relative to the testing machine can be seen in Figure 2. Additionally, the DIC measurements were compared with strain gauges measurements. The SG positions can be seen in the Figure 1.

The commercial DIC system ARAMIS 4M high resolution (HR) has been applied in this work and is suited for measurements of three-dimensional deformations and 2D surface strains. The results can through the ARAMIS post-processing software be presented in terms of various full-field illustrations of deformation in the specimen. The measurement frequency was set to $1 \mathrm{~Hz}$ making it possible to store images directly onto the hard drive of the system computer.

The ARAMIS high speed system (HS) consists of two external Photron APX-RS high-speed cameras with a maximum data acquisition rate of $250.000 \mathrm{fps}$ and $3.000 \mathrm{fps}$ at full resolution (1 MPixel). However, in order to maximize the measurement frequency the image resolution was adjusted to the specimen size, resulting in a resolution of $128 \times 1024$ pixels and a measurement frequency of $10.000 \mathrm{fps}$.

The HS system was used in a parallel setup with the HR system in order to detect the initial failure location and crack propagation rate during the brittle failure mechanism, where an interface crack is propagating between the straps and the inner adherent.

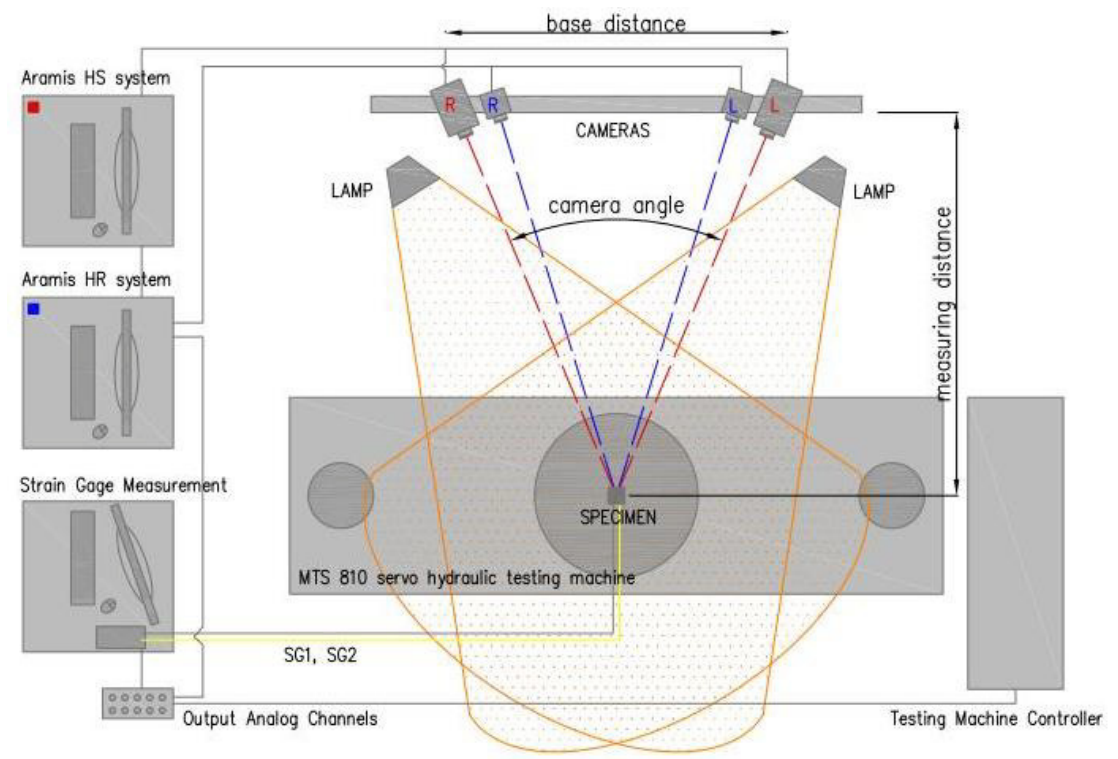

Fig. 2. Schematic representation of the test setup and measurement equipment positioning.

\subsection{DIC Setup}

\subsubsection{Sensor Setup}

In order to apply a suitable DIC speckle pattern, black paint is applied from a spray can by softly pressing the spray button so that an irregular contrast speckle pattern is applied. The speckles applied should be of adequately small size so that multiple grayscales are contained within each facet of the 
computational mask. The spatter marks should generally be covered by $3 \times 3$ to $6 \times 6$ pixels. This guideline is independent of the sensor size and therefore usable across the two applied DIC systems (and chosen resolution for the HS system) [3].

The cameras are placed at the measuring distance, base distance and camera angle defined in Figure 2 and listed in Table 1, according to the used lens focal length as well as sensor configuration $[3]$.

Table 2. 3D DIC systems setup

\begin{tabular}{|l|l|c|}
\hline Setting & HR & HS \\
\hline
\end{tabular}

\begin{tabular}{|l|c|c|}
\hline base distance & $210-380 \mathrm{~mm}$ & $410-580 \mathrm{~mm}$ \\
\hline measuring distance & $500-700 \mathrm{~mm}$ & $500-700 \mathrm{~mm}$ \\
\hline focal length & $50 \mathrm{~mm}$ & $50 \mathrm{~mm}$ \\
\hline Aperture & 11 & 2,8 \\
\hline frame/sec (fps) & 1 & 10.000 \\
\hline Shutter time & $25 \mathrm{~ms}$ & $1 / 10000$ \\
\hline calibration panel & $80 \times 100,250 \times 250 \mathrm{~mm}$ & $80 \times 100,250 \times 250 \mathrm{~mm}$ \\
\hline
\end{tabular}

In order to achieve adequate lighting, a 500W halogen spot lamp was placed slightly higher than the cameras. Owing to the fact that the HS system requires a large amount of illumination, while for the HR system too much illumination will cause overexposed images, circular standard $50 \mathrm{~mm}$ polarizing filters were applied on the HR camera lenses and thus a coordinated illumination setup for both types of cameras became possible and was established. The aperture settings for the HS system cameras were set as widely open, and keeping the shutter time as low as possible to minimize blur. However, the crack flank and total specimen movement during crack propagation, which is of interest in this work, are expected to be very small. Therefore, the shutter time can be relaxed if needed. Finally, it is important to notice that the only reason for using a 3D DIC setup instead of a 2D setup is because both HS and HR camera sets cannot be placed perpendicular to the same surface. All the adjustments for both camera systems applied during the testing are shown in Table 1.

\subsubsection{Calibration}

Prior to the recording of the images, the cameras are calibrated using a certified calibration panel. The calibration object is determined by the measuring volume. The size of the calibration panel was chosen according to the sensor configuration shown in Table 1. The standard calibration of the HR system was carried out automatically guided by the ARAMIS system software. However, the calibration of HS system, due to the external high speed cameras, must be done manually feeding a series of individually recorded images into the ARAMIS software for calibration. The details of this calibration procedure are outside the scope of this paper, but can be found in [3]. .

However, for both the HR and HS pixel calibration deviations less than 0.04 pixels were obtained.

\section{Results}

\subsection{DSL specimen results}

Table 2 lists measured failure loads and strains for all specimens where DIC measurements were carried out. Additionally, Figure 3 shows the trend of the failure load versus overlap length and material. 
Table 2. DSL specimen results for specimens where DIC measurements were carried out. Failure loads and max. major strains are obtained from both DIC and strain gauge measurements at the SG2 location, see Fig. 1.

\begin{tabular}{|c|c|c|c|c|c|c|}
\hline Specimen & $\begin{array}{c}\text { Overlap } \\
{[\mathrm{mm}]}\end{array}$ & $\begin{array}{c}\text { Strap } \\
{[\mathrm{mm}]}\end{array}$ & $\begin{array}{c}\text { Strap } \\
\text { material }\end{array}$ & $\begin{array}{c}\text { failure load } \\
{[\mathrm{kN}]}\end{array}$ & $\begin{array}{c}\text { DIC: } \\
\text { max. major } \\
\text { strain [\%] }\end{array}$ & $\begin{array}{c}\text { SG: } \\
\text { max. major } \\
\text { strain [\%] }\end{array}$ \\
\hline
\end{tabular}

\begin{tabular}{|c|c|c|c|c|c|c|}
\hline 100/3UH/D2.1 & 100 & 3 & UH & 68,25 & 0,26 & 0,93 \\
\hline 100/3UH/D2.2 & 100 & 3 & UH & 67,61 & 0,72 & 0,82 \\
\hline 100/3UH/D2.3 & 100 & 3 & UH & 65,78 & 0,72 & 0,73 \\
\hline $150 / 3 \mathrm{UH} / \mathrm{D} 3.1$ & 150 & 3 & $\mathrm{UH}$ & 70,27 & 0,42 & 0,57 \\
\hline 150/3UH/D3.2 & 150 & 3 & $\mathrm{UH}$ & 64,95 & 0,15 & 0,60 \\
\hline $200 / 3 \mathrm{UH} / \mathrm{D} 4.1$ & 200 & 3 & $\mathrm{UH}$ & 66,55 & 0,22 & 0,69 \\
\hline $200 / 3 \mathrm{UH} / \mathrm{D} 4.3$ & 200 & 3 & $\mathrm{UH}$ & 66,94 & 0,29 & 0,50 \\
\hline 100/6UH/D6.2 & 100 & 6 & $\mathrm{UH}$ & 59,04 & 0,17 & 0,66 \\
\hline $100 / 6 \mathrm{UH} / \mathrm{D} 6.3$ & 100 & 6 & $\mathrm{UH}$ & 72,12 & 0,25 & 0,92 \\
\hline $150 / 6 \mathrm{UH} / \mathrm{D} 7.1$ & 150 & 6 & $\mathrm{UH}$ & 67,71 & 0,30 & 0,60 \\
\hline 150/6UH/D7.2 & 150 & 6 & $\mathrm{UH}$ & 58,70 & 0,21 & 0,55 \\
\hline $200 / 6 \mathrm{UH} / \mathrm{D} 8.1$ & 200 & 6 & $\mathrm{UH}$ & 89,50 & 0,43 & 1,60 \\
\hline $200 / 6 \mathrm{UH} / \mathrm{D} 8.3$ & 200 & 6 & $\mathrm{UH}$ & 74,27 & 0,22 & 0,92 \\
\hline $200 / 6 \mathrm{UH} / \mathrm{D} 9.1$ & 200 & $6^{*}$ & $\mathrm{UH}$ & 104,04 & 0,52 & 0,56 \\
\hline $200 / 6 \mathrm{UH} / \mathrm{D} 9.2$ & 200 & $6^{*}$ & $\mathrm{UH}$ & 90,41 & 0,33 & 0,47 \\
\hline
\end{tabular}

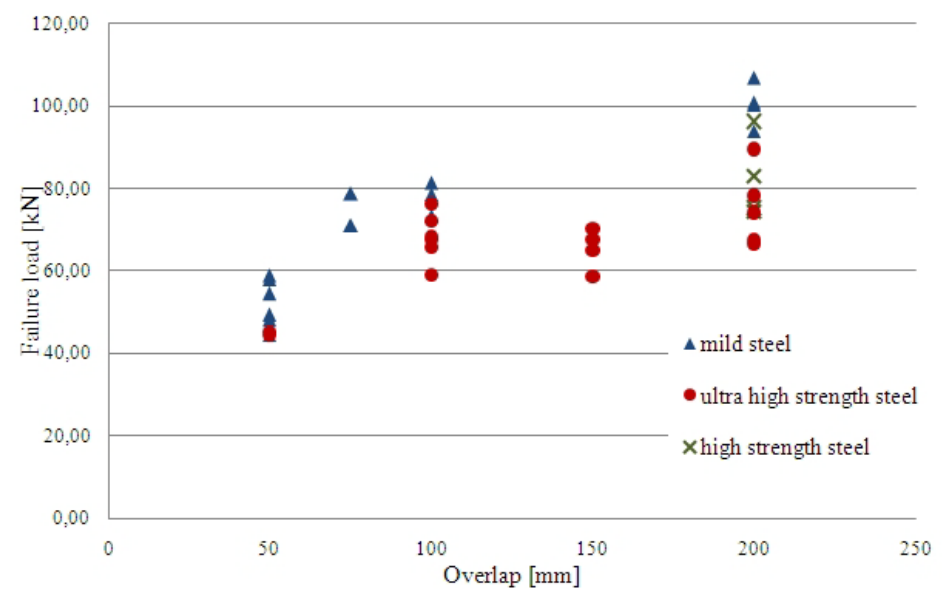

Fig. 3. Failure load versus overlap length for all tested specimens.

\section{Comparison and Discussion}

\subsection{DSL joints results}

From the experimental results (Figure 3 and Table 2) the following observations can be drawn:

1. Generally the ultra high strength (UH) steel specimens with $6 \mathrm{~mm}$ strap thickness at the same overlap length fail at around 10\% higher load level than the similar $3 \mathrm{~mm}$ strap thickness specimens, except for the $150 \mathrm{~mm}$ overlap specimens. However, variations are sufficiently small to be addressed to specimen manufacturing variations. 
2. UH steel specimens with the same strap thickness but with large overlap lengths fail at around the same loads, indicating that only a limited static strength advantage over $100 \mathrm{~mm}$ overlap can be achieved.

3. The high strength steel joints show on average a $20 \%$ lower capacity in comparison with the equivalent joints with mild steel. This indicates that yielding in the mild steel specimens redistributes the stresses resulting in a higher overall capacity of the joint.

4. The UH steel joints show on average a $10 \%$ lower strength in comparison with the equivalent joints with mild steel and an approximately the same strength in comparison with the equivalent joints with high strength steel. The results so far show that the use of ultra high strength steel/high strength steel could be a disadvantage when other practical design and operational issues are neglected.

\subsection{Strain measurements}

The strain gauge data logged from SG2 (see Figure 1) were compared with DIC measurements at approximately the same vertical location (see Figure 4). The SG strain data was measured at $5 \mathrm{~mm}$ distance from the end of the strap on the front side of the specimen. The DIC strain data was recorded on the lateral edge side of the specimen (see Figures 1 and 4). Preferably, the measurements should have been taken in the same position, but it was prioritized to be able to monitor the crack propagation on the lateral edge side of the specimen with the DIC-systems, thus making an exact comparison of strains measured with DIC and strain gauges impossible. Therefore, the measured strains will be different due to the local strain distributions at the SG2 position. However, independently measured strains from the HR and HS DIC systems show the same strain distribution as well as maximal strain values verifying the measured DIC strain measurements against each other.

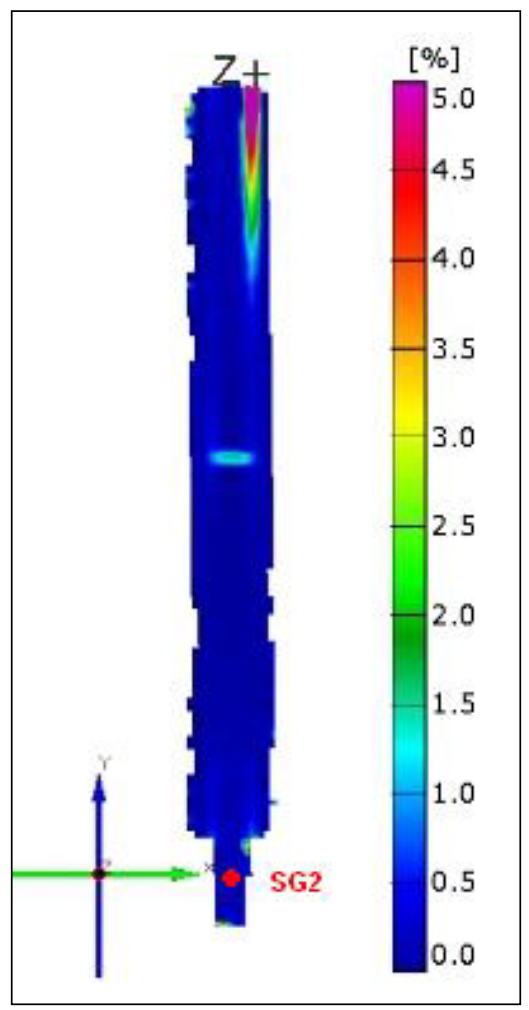

Fig. 4. Specimen 100/6UH/D6.3. The contour plot shows the equivalent strain distribution [\%] measured with the HR DIC-system based on the last captured frame prior to the final failure moment. 

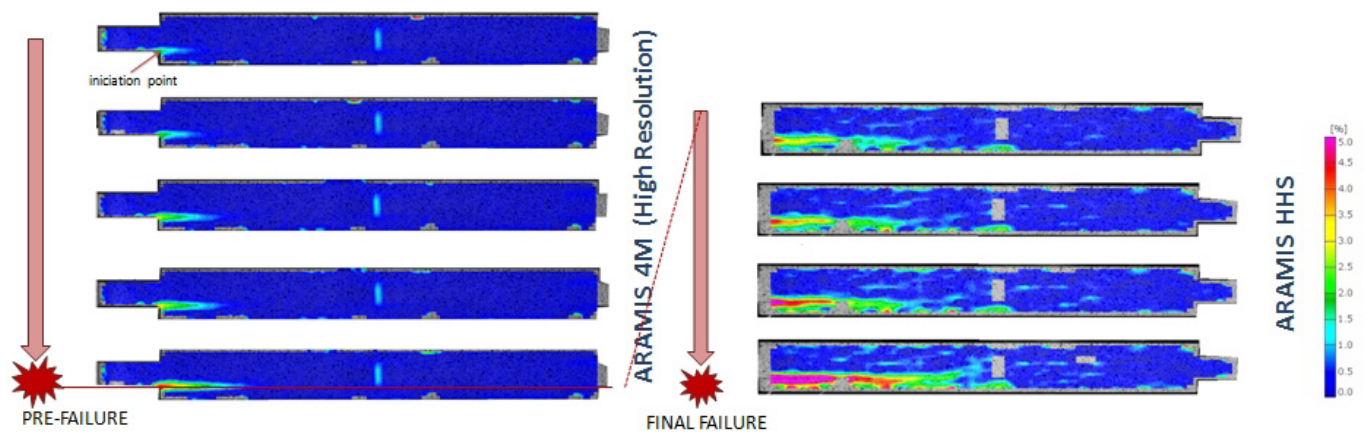

Fig. 5. Specimen 100/6UH/D6.2 results. The HS system detects where the failure initiates and how the interface crack propagates, while the HR system detects the pre-failure deformations in the specimen.

As has been mentioned earlier, the main reason for applying a parallel setup with both a HR and HS system is the opportunity to provide a better understanding of the DSL joint failure evolution and final failure mechanisms. The HR system provided an overall view on the entire uploading regime and indicated from where the final failure in terms of rapid crack propagation would start, see Figure 5. The HS system captured first of all the rapid brittle crack growth mechanism itself, illustrated by artificially high strains over the crack flanks, making it possible to identify the position of the instant crack tip, see Figure 5. Secondly, the HS system was used to verify the strain measurements of the HR system at the initial overlapping loading stages prior to final failure captured by both systems (the HS cameras operate in a ring buffer storing only images from a limited time period before final failure).

The crack initiation and propagation data from the HR and HS systems respectively show a trend (with the exception of UH $3 \mathrm{~mm}$ steel strap specimens) where the crack initiates at the outer edges of the DSL joint, confirming earlier analytical and numerical investigations of the DSL-specimen $[1,4,5]$. The combined stiffness of the outer adherends is larger than that of the inner adherend, such that the largest load transfer happens through the bondline at the free ends of the outer adherends, i.e. the crack propagation initiates where the critical peel and shear stresses appear from a theoretical point of view $[1,4,5]$. Furthermore, the data captured from the HR system through the uploading regime indicates that the straps on both sides of the loaded adherend are not loaded exactly equally indicating that manufacturing misalignments are present. The latter effect can have an influence on the crack propagation initiation load level.

\section{Conclusion}

In order to gain a better understanding of the failure mechanisms at play for DSL specimens, two 3D DIC-systems (used in parallel), were used for the deformation analysis of the specimen surface and for determining the fracture behavior including the crack propagation happening rapidly at the failure moment. A mutual DIC test setup was designed making it possible to operate both systems in parallel.

The results from the HS system showed that the failure process tend to initiate at the outer edges of the DSL joint where the combined stiffness of the outer adherends is larger than that of the inner adherend, such that the largest load transfer happens through the bondline at the free ends of the outer adherends. The HR system furthermore confirmed this trend.

It was not possible to directly compare the strain measurements from the HS and HR systems with strain gauge data. However, it was possible to verify the measured DIC strain data between the two systems and good agreement was found.

The dual 3D DIC-system application for the present DSL experimental testing proved to be a powerful tool to provide the strain and displacement data for a test configuration where both the 
uploading regime and a rapid final failure mechanism is at play. The setup furthermore made it possible to detect both the initial failure location and the crack propagation rate during the brittle failure mechanism.

\section{References}

1. Hashim, S., Nisar, J., Tsouvalis, N. J., Anyfantis, K., Moore, P., Chirica, I., Berggreen, C., Orsolini, A., Quispitupa, A., McGeorge, D., Hayman, B., Boyd, S., Misirlis, K., Downes, J., Dow, R. S. and Juin, E., Fabrication, Testing and Analysis of Steel/Composite DLS Adhesive Joints, MARSTRUCT 2nd International Conference on Marine Structures, Lisbon, Portugal, March 16-18, (2009)

2. Hashim, S. A. and Knox, E. M., Aspects of joint design and evaluation in thick-adherend applications, The Journal of Adhesion,80:7, 569-583 (2004)

3. GOM Optical Measuring Techniques. Aramis v5.4 User Manual, GOM mbH (2005)

4. Osnes, H., McGeorge, D., Experimental and analytical strength analysis of double-lap joints for marine applications. Composites: Part B 40, 29-40 (2009)

5. Hashim, S., Berggreen, C., Tsouvalis, N., McGeorge, D., Chirica, I., Boyd, S., Moore, P., Juin, E., Nisar, J., Anyfantis, K. and Misirlis, K., Design and Analysis of DLS Steel/Composite Thick-Adherend Adhesive Joints, 17th International Conference on Composite Materials (ICCM-17), Edinburgh, UK, July 27-31, (2009)

6. Adams, R.D., Comyn, J.,Wake, W.C., Structural adhesive joints in engineering, (Chapman \& Hall Publisher, London, UK, 1997)

7. da Silva L.F.M., Neves P., Adams R.D, Spelt J.K. Analytical models of adhesively bonded joint-Part I: literature survey, in J. Adhesion and Adhesives 29, 319-330(2009).

8. Grabovac I. Bonded composite solution to ship reinforcement. Composite A 34, 847-854 (2003).

9. Khodorkovsky, Mouring, S., Shkolnikov. Advanced hybrid joining technology, Proc. ARANET Lightweight Marine Structures, Glasgow 2009 